\title{
CAPÍTULO 26: MINI-REVISÃO FOCADA NAS APLICAÇÕES BIOTECNOLÓGICAS DA QUITOSANA: EMBALAGENS, ALIMENTOS \& BEBIDAS
}

\section{CHAPTER 26: MINI-REVIEW FOCUSED ON THE BIOTECHNOLOGICAL APPLICATIONS OF CHITOSAN: PACKAGING, FOOD \& BEVERAGE}

\author{
Vagne de Melo Oliveira ${ }^{1}$; Márcia Nieves Carneiro da Cunha² ${ }^{2}$ Juanize Matias da Silva Batista ${ }^{3}$, \\ Beatriz de Aquino Marques da Costa ${ }^{4}$ e Ana Lúcia Figueiredo Porto ${ }^{5}$
}

\begin{abstract}
Resumo
A quitosana é um polissacarídeo obtido da quitina e que tem sido utilizado em processos ambientais, agrícolas, terapêuticos e industriais. Dentre estes, a indústria alimentícia parece ser a que melhor explora essa matéria-prima, visando à fabricação de embalagens, preparação de alimentos, ou mesmo como aditivos em bebidas. Assim, este trabalho objetivou revisar brevemente sobre as aplicações biotecnológicas da quitosana na indústria alimentícia. Para tanto, foram utilizadas buscas em bases científicas para a realização do levantamento bibliográfico (ScienceDirect, Elsevier, Scopus, Springer, TandFonline, PubMed, Wiley Online Library e Google Scholar). Foi identificado grande volume de publicações, sinalizando a importância do tema para a área industrial, sendo diagnosticadas a partir das publicações disponíveis que o uso da quitosana está diretamente relacionado às suas propriedades (biodegradabilidade, biocompatibilidade, atoxicidade) e que deficiências ocasionais podem ser supridas associando este polímero a outros compostos naturais. $\mathrm{O}$ volume científico trata de aplicações em processos de águas residuais, clarificação e aditivos de bebidas, aumento do tempo de prateleira dos alimentos, proteção contra microrganismos deteriorantes e formação de filmes biodegradáveis para revestimento de frutas e vegetais. Por fim, as fontes aquáticas têm recebido especial atenção pela sua abundância e facilidade de extração, além de apresentar funções biológicas que são desejadas pela indústria nutracêutica.
\end{abstract}

Palavras-Chave: alimentos, bebidas, embalagens, nutracêuticos.

\begin{abstract}
Chitosan is a polysaccharide obtained from chitin and it has been used in environmental, agricultural, therapeutic, and industrial processes. Among these, the food industry seems to be the one that exploits this raw material the best, aiming at package manufacturing, food preparation, or even as beverage additives. Thus, this work aimed to briefly review the biotechnological applications of chitosan in the food industry. For that, searches in scientific bases were used to carry out the bibliographic survey (ScienceDirect, Elsevier, Scopus, Springer, TandFonline, PubMed, Wiley Online Library and Google Scholar). A large volume of publications was identified, signaling the importance of the topic for the industrial area, from the available publications, it was detected that the use of chitosan is directly related to its properties (biodegradability, biocompatibility, atoxicity) and that occasional deficiencies can be addressed by associating this polymer to other natural compounds. The scientific volume deals with applications in waste water processes, clarification and beverage additives, increased shelf life of food, protection against deteriorating microorganisms and formation of

\footnotetext{
${ }^{1}$ Doutor, Universidade Federal Rural de Pernambuco, vagne_melo@ hotmail.com

${ }^{2}$ Doutora, Universidade Federal Rural de Pernambuco, marcianieves@ yahoo.com.br

${ }^{3}$ Doutora, Universidade Federal Rural de Pernambuco, juanizematias@ yahoo.com.br

${ }^{4}$ Mestranda em Biociência Animal, Universidade Federal Rural de Pernambuco, deaquinobeatriz@gmail.com

${ }^{5}$ Professora Titular, Universidade Federal Rural de Pernambuco, analuporto@ yahoo.com.br
} 
biodegradable films for coating fruits and vegetables. Finally, aquatic sources have received special attention for their abundance and ease of extraction, in addition to presenting biological functions that are desired by the nutraceutical industry.

Keywords: food, beverages, packaging, nutraceuticals.

\section{Introdução}

Polímeros são compostos formados por monômeros unidos, covalentemente, numa reação de polimerização (OLATUNJI, 2020). Os biopolímeros são polímeros produzidos por organismos vivos, enquanto que biopolímero aquático é um termo que faz referência aos polímeros provenientes de ambiente dulcícola e/ou marinho (OLIVEIRA et al., 2021). Biopolímeros aquáticos são de extremo interesse industrial e médico devido as suas propriedades bioativas e de capacidade para formação de biomateriais, destacando-se o colágeno, alginato, pectina, celulose, agarose, ágar, carragenina, gelatina, dextrana, xantana, quitina, além de outros polímeros, que têm recebido especial atenção nos últimos anos devido à sua abundância, biodisponibilidade, alta versatilidade, baixa toxicidade, boa biocompatibilidade, biodegradabilidade, flexibilidade, entre outros, oferecendo aplicações multifuncionais para no âmbito industrial (EL KNIDRI et al., 2018; HU et al., 2018; MARTĂU; MIHAI; VODNAR, 2019; SHARIATINIA et al., 2019; OLIVEIRA et al., 2021).

A crescente atenção para a utilização, ou mesmo reutilização, dos biopolímeros aquáticos está ligada principalmente as questões ambientais, uma vez que muitos desses polímeros são descartados (AHMED et al., 2018). A quitina é um dos biopolímeros mais abundantes na natureza (EL KNIDRI et al., 2018), podendo ser obtido a partir de insetos, fungos (MUANPRASAT; CHATSUDTHIPONG, 2017; VERLEE; MINCKE; STEVENS, 2017), moluscos e exoesqueletos de crustáceos, sendo esta o principal recurso para obtenção desse polímero, principalmente através da carapaça dos crustáceos (MANIGANDAN et al., 2018). Esse biopolímero pode ser extraído de organismos aquáticos por método químico e biológico. O primeiro é o mais utilizado devido à rapidez do processo de extração, porém é oneroso e considerado ambientalmente prejudicial, enquanto o segundo método é o menos utilizado, mas tem se destacado pelo alto rendimento obtido nas extrações, principalmente quando a fonte é aquática, como camarões (PRIYADARSHI; RHIM, 2020).

A quitina extraída pelo método químico ou biológico é convertida em quitosana, um derivado polissacarídeo policatiônico natural (PAUL; KOLESINSKA; SUJKA, 2019), considerado um copolímero linear de 2-acetamido-2-desoxi-D-glicopiranose e 2-amino-2desoxi-D-glicopiranose ligada a $\beta$-(1 $\rightarrow 4)$ (AHMED et al., 2018; SHARIATINIA, 2019), através do processo bioquímico de desacetilação, envolvendo a remoção de grupos acetil 
$\left(\mathrm{COCH}_{3}^{-}\right)$da estrutura da quitina. Esta $\mathrm{N}$-desacetilação nunca é completa e, portanto, são obtidos quitosana com diferentes graus de desacetilação. A desacetilação da quitina pode ser realizada através de dois métodos: alcalino e enzimático (PRIYADARSHI; RHIM, 2020), consumindo tempo, energia e reagentes (EL KNIDRI et al., 2018). O grupo amina primário da quitosana é responsável por suas propriedades, como a natureza catiônica, entrega controlada de medicamentos e compostos ativos, muco-adesão, antimicrobiana, antioxidante, antifúngica, aprimoramento da permeação, entre outros (ELSABEE; ABDOU, 2013; ALI; AHMED, 2018; PRIYADARSHI; RHIM, 2020). Além disso, a quitosana possui vários grupos funcionais $\mathrm{OH}$ e $\mathrm{NH}_{2}$ que permitem sua ligação às proteínas e íons metálicos (SHARIATINIA, 2019).

Biopolímeros de quitosana têm sido amplamente utilizados em ambientes alimentícios, biomédicos, cosméticos, farmacêuticos e nutracêuticos devido à sua origem natural e inúmeras propriedades funcionais (PATRULEA et al., 2015; MUXIKA et al., 2017; AHSAN et al., 2018; EL KNIDRI et al., 2018; BARANWAL et al., 2018; KRAVANJA et al., 2019; MU et al., 2019; SHARIATINIA et al., 2019; WANG et al., 2020). Dentro dos diferentes segmentos comerciais, a indústria de alimentos tem cada vez mais incentivado prospecções para uso de biopolímeros de quitosana na preparação de microesferas visando o encapsulamento de bioativos para fins nutracêuticos (AKBARI-ALAVIJEH; SHADDEL, R.; JAFARI, 2020), ou mesmo atuar como agente estabilizante e espessante, e até para servir de matéria-prima para confecção de embalagens para proteção e prolongamento da vida útil de prateleira de frutas e vegetais, algumas das quais até podendo ser comestíveis (MARTĂU; MIHAI; VODNAR, 2019). Diante deste cenário, este trabalho objetivou realizar uma breve revisão sobre as principais aplicações biotecnológicas dos biopolímeros de quitosana na indústria alimentícia, focando, primordialmente, nos segmentos de consumo alimentícios, bebidas e de embalagens.

\section{Desenvolvimento}

Esta mini-revisão foi desenvolvida a partir de levantamento bibliográfico, fazendo uso de plataformas eletrônicas científicas, como: ScienceDirect (https://www.sciencedirect.com/), Elsevier (https://www.elsevier.com/), TandFonline (https://www.tandfonline.com/), Scopus (https://www.scopus.com/), Google Scholar (https://scholar.google.com.br/), Springer (https://www.springer.com/), PubMed (https://pubmed.ncbi.nlm.nih.gov/) e Wiley Online Library (https://onlinelibrary.wiley.com/), utilizando como descritores as palavras-chave: quitosana, quitosana em alimentos, quitosana em bebidas, quitosana em embalagens, 
quitosana e aplicações. Realizou-se a leitura dos títulos e resumos para seleção dos artigos, livros, capítulos de livro, notas científicas, entre outros, excluindo os que não estavam dentro do escopo da proposta ou mesmo que não detinham informações relevantes para nossa investigação. Foram incluídas publicações em inglês e português que atenderam aos critérios de busca.

\section{Visão geral}

A indústria de Aquicultura é um dos ramos comerciais que estão em evidência pelo crescimento e participação na economia global. Como um subproduto principal da indústria de pescado e frutos do mar, uma grande quantidade de resíduos de carapaças de crustáceos é gerada a cada ano, atrelada a esse crescimento, com potencial para ser usada para produzir quitina de valor agregado e esta ser convertida em quitosana através da desacetilação. Sendo extraído de um resíduo orgânico usando muitos métodos de eficiência energética, a obtenção da quitosana se torna menos onerosa quando comparada a obtenção de polímeros sintéticos (PRIYADARSHI; RHIM, 2020). Moléculas de quitosana apresentam uma ampla gama de propriedades úteis, incluindo atividades antimicrobianas e antiinflamatórias. A produção química de oligômeros de quitosana parcialmente acetilados é prejudicial ao meio ambiente e é difícil controlar o grau de polimerização e acetilação. Esses problemas podem ser resolvidos com o uso de enzimas específicas (quitinases, quitosanases, quitina desacetilases) que auxiliam na produção de quitosana da melhor forma (KIDIBULE et al., 2018; SCHMITZ et al., 2019). Assim, as propriedades físico-químicas e bioativas da quitosana fazem dessa matéria-prima uma opção viável e promissora para uso em preparações alimentícias, produção de bebidas e fabricação de embalagens (PRIYADARSHI; RHIM, 2020).

\section{Aplicações biotecnológicas da quitosana}

As aplicações biotecnológicas da quitosana se estendem por inúmeras áreas, tais como ambiental, industrial, agrícola e médica (CHEBA, 2020). Biopolímeros de quitosana, seja nativo ou modificado, são adequados para uso na indústria alimentícia devida suas excelentes propriedades (biodegradabilidade, biocompatibilidade, atoxicidade) (CHEUNG et al., 2015; DRAGOSTIN et al., 2016; FONSECA-SANTOS; CHORILLI, 2017; XU et al., 2018; ZHAO et al., 2018; MATICA et al., 2019; PAUL; KOLESINSKA; SUJKA, 2019), para serem aplicado em processos de purificação de água, clarificação e desacidificação de sucos, extensão de vida útil de alimentos, retardo da deterioração microbiana, melhoria da qualidade de alimentos, aditivos alimentícios e para formação de filmes para embalagens biodegradáveis (CHEBA, 2020). 
A quitosana pode ser fornecida pelo mercado comercial na forma de fibras, filmes, géis, esponjas e/ou nanopartículas (DUTTA; TRIPATHI; DUTTA, 2012; ROZMANET al., 2019; OTTONELLI et al., 2020; SATO et al., 2020), podendo ser utilizada para o tratamento de água residual (SARODE et al., 2019), fabricação de papel (SONG et al., 2018), agricultura (KASHYAP; XIANG; HEIDEN, 2015), na promoção do desempenho reprodutivo de peixes cultiváveis (BHAT et al., 2018), na área de engenharia óssea (GAIHRE; LECKA-CZERNIK; JAYASURIYA, 2018), odontológica (AGUILAR et al., 2019), na entrega de genes (CAO et al., 2019), tratamento de fibrose hepática (WANG et al. 2018), biomédica, apresentando ação antiplaquetária, anticoagulante (SONIN et al., 2020), antiinflamatória, anti-hipertensiva (MUANPRASAT; CHATSUDTHIPONG, 2017), antifúngica (LIU et al., 2018), neuroprotetora (CHEN; LI; BORGENS, 2018), promotora da regeneração de tecidos epiteliais (DRAGOSTIN et al., 2016; IACOB et al., 2018), nanofibras para entrega de insulina (LANCINA; SHANKAR; YANG, 2017), empregada na terapia de medicamentos para tratamento do câncer (YOUNES et al., 2012; BABU; RAMESH, 2017; DRAGOSTIN et al., 2020), adjuvante para entrega de vacinas (ELIEH-ALI-KOMI; HAMBLIN, 2016; MALIK et al., 2018), integrante de formulações cosméticas e de usos têxteis, químicos, fotográficos, cromatográficos, eletrônicos, na fabricação de papeis e, especialmente, nos segmentos relacionados a indústria de consumo de alimentos, bebidas (ROCHA; COIMBRA; NUNES, 2017) e, principalmente, na fabricação de embalagens para produtos de origem animal e de origem vegetal, como de frutas e leguminosas (PRIYADARSHI; RHIM, 2020).

A quitosana tem sido relatada como excelente componente bioativo para fins nutracêuticos, como descrito por Wang et al. (2019) ao investigarem o potencial termogênico da quitosana e de oligossacarídeo de quitosana no tratamento da obesidade em ratos. Segundo os autores, ambos os polissacarídeos contribuíram para redução do ganho de peso em ratos obesos induzidos por dieta rica em gordura, sem afetar a ingestão de alimentos, e inibiram efetivamente a hipertrofia e hiperplasia do tecido adiposo, além de aumentarem a expressão de genes e proteínas de escurecimento (UCP1, PGC1 $\alpha$, PRMD16, e ATF2) no tecido adiposo branco e no marrom. Também, hidrogéis à base de quitosana têm sido amplamente estudados devido à sua preparação simples, boa biocompatibilidade e reparabilidade em condições fisiológicas para administração (XU et al., 2019). Fan et al. (2020) desenvolveram hidrogéis preparados a partir de colágeno reticulado de pele de tilápia (Oreochromis niloticus) e quitosana para entrega de nanocorpos. Os resultados indicaram a viabilidade da quitosana na entrega de medicamentos, potencializando sua utilização para usos na terapia do câncer. 
A quitosana apresenta boa atividade antimicrobiana frente uma grande variedade de microrganismos patogênicos e deteriorantes, principalmente, de alimentos, incluindo fungos e bactérias Gram+ e Gram- (DUTTA; TRIPATHI; DUTTA, 2012). Samayanpaulraj et al. (2020) investigaram o potencial antibacteriano da quitosana de camarão (Penaeus indicus) frente a isolados de Aeromonas hydrophila virulenta Ah17 e observaram que ela foi capaz de controlar o crescimento dessa cepa. Nanopartículas de quitosana também já foram reportadas com ação antimicrobiana frente a uma gama de bactérias, fungos, leveduras e algas, através de estudos in vivo quanto in vitro, sendo utilizado como revestimento antimicrobiano, em processos cicatriciais (feridas, queimaduras), na prevenção de doenças infecciosas e na inibição de agentes deteriorantes de alimentos, especialmente, frutas (ROZMANET al., 2019).

Daetz et al. (2016) e Oliveira et al. (2020) utilizaram micropartículas de quitosana para tratar metrite em vacas leiteiras em período de lactação. As nanofibras à base de quitosana têm uma proporção de área de superfície: volume maior do que os materiais a granel em escala macro. Além disso, nanofibras à base de quitosana podem levar a porosidade e propriedades mecânicas aumentadas, melhorando, assim, as funcionalidades da superfície e, consequentemente, a gama de aplicações. No entanto, a diversidade de fontes de matériaprima e o tipo de processo utilizado para extração da quitina e produção da quitosana podem fornecer características e propriedades físico-químicas distintas (FARIAS; SANT'ANNA CADAVAL JUNIOR; ALMEIDA PINTO, 2019). Também, ressalta-se que, o exame de toxicidade desses biopolímeros e de seus derivados é um procedimento essencial para avaliar a possibilidade de usar essas concentrações como ingrediente alimentar (DARWESH et al., 2018).

\section{Aplicação biotecnológica: filmes/embalagens/revestimentos}

O processo de embalagem de alimentos deve ser feito de forma criteriosa quando se trata da preservação de alimentos frescos ou processados. Ele desempenha um papel importante em fazer com que o alimento, que é preparado em um local, esteja disponível para os consumidores em algum outro lugar após dias, semanas ou mesmo meses após a colheita ou fabricação inicial (PRIYADARSHI; RHIM, 2020). Nesse sentido, compósitos à base de quitosana são opções viáveis para a fabricação de embalagens, principalmente, destinadas a indústria alimentícia, podendo ser utilizados como filmes e/ou revestimentos comestíveis (SOUZA et al., 2020). Levando em consideração a abundância dos biopolímeros aquáticos em fornecer quitina, os materiais derivados têm sido amplamente aplicados em vários campos ligados ao ambiente alimentar, muito devido suas propriedades biológicas, como capacidade 
antioxidante e sua disposição para a formação de filmes (WANG; QIAN; DING, 2018; HAMIL et al., 2020).

Em embalagens destinadas a utilização em alimentos, os efeitos antimicrobianos se somam às propriedades de barreira dos materiais, para aumentar a vida útil e a qualidade dos produtos. A quitosana é um polissacarídeo bioativo natural com atividade antimicrobiana intrínseca e, devido às suas propriedades físico-químicas excepcionais transmitidas pela estrutura do polissacarídeo, foi reconhecida como uma alternativa natural aos polímeros antimicrobianos sintetizados quimicamente. Este, associado à crescente preferência por materiais biofuncionais de recursos renováveis, resultou em um interesse significativo sobre o potencial de aproveitamento de quitosana em materiais de embalagem (VAN DEN BROEK et al., 2015).

Para aplicações em embalagens de alimentos, a quitosana pode ser usada na forma de filmes de embalagem ou revestimentos diretamente no material alimentar (PRIYADARSHI; RHIM, 2020). Hamil et al. (2020) utilizaram as estruturas espinhosas de ouriço-do-mar (Paracentrotus lividus), que geralmente são encontradas em toneladas de mortos à beira-mar, associada com gel de quitosana para produção de filmes biodegradáveis e observaram uma melhora da hidrofobicidade dos filmes, além do que, a presença da quitosana foi capaz de aumentar a estabilidade antioxidante e térmica dos filmes, tornado-se uma alternativa para produção de filmes hidrofóbicos e de alta estabilidade térmica.

As fracas propriedades mecânicas, permeabilidade a gases e vapor d'água limitam o uso da quitosana (ELSABEE; ABDOU, 2013) e isso tem proporcionado uma quantidade significativa de investigações usando a quitosana de modo associativo a outros polímeros, como alginato, celulose e colágeno, por exemplo. Para melhorar os atributos biológicos e fisiológicos, principalmente mecânicos, térmicos e de barreira, dos filmes à base de quitosana, uma vasta mistura de extratos de plantas e polímeros de suporte foi misturada em filmes de quitosana (MUJTABA et al., 2019), obtendo-se excelente resultados.

Andrade et al. (2019) investigaram o efeito da incorporação de nanocelulose obtida de diferentes fibras (espiga de milho, casca de milho, casca de coco e farelo de trigo) aos filmes de quitosana/glicerol e observaram que a fonte da nanofibras foi determinante na definição das propriedades dos filmes de quitosana/glicerol formados, apresentando-se promissor para o desenvolvimento de novos filmes biodegradáveis para revestimento. Pereda et al. (2014) prepararam filmes polieletrolíticos concebidos como embalagens potencialmente comestíveis para alimentos preparados por moldagem de emulsões formadoras de filmes à base de quitosana, glicerol e azeite, contendo nanocristais de celulose dispersos. Segundo dados da 
pesquisa, o uso combinado de nanopartículas de celulose e azeite de oliva provou ser um método eficiente para reduzir a permeabilidade elevada ao vapor de água dos filmes de quitosana plastificados, melhorando ainda seu comportamento à tração. Os testes mecânicos revelaram que todos os filmes analisados apresentaram dois relaxamentos principais atribuídos às fases ricas em glicerol e quitosana, respectivamente.

Ren et al. (2017) examinaram a influência da concentração do polímero de quitosana nas propriedades mecânicas e de barreira de filmes de amido de milho/quitosana. A pesquisa comprovou que a incorporação de quitosana resultou no aumento da solubilidade do filme, em diferenças totais de cor, na resistência à tração e na diminuição na permeabilidade ao vapor de água. Segundo os autores, os resultados são indicativos da real possibilidade de produção de filmes biodegradáveis para serem utilizadas em alimentos e medicamentos a partir da quitosana associada a compostos vegetais.

Suseno et al. (2014) investigaram o aumento da vida útil da banana Cavendish usando o revestimento comestível de quitosana. Os autores reportaram que a banana revestida pelo filme demonstrou atrasos no processo de amadurecimento em comparação com a banana não revestida, além das constatações da redução na perda de peso, bem como na perda de vitamina $\mathrm{C}$ em comparação com a banana não revestida. A perda de peso e a de vitamina $\mathrm{C}$ diminuiu com o aumento da concentração de quitosana e o grau de desacetilação dela.

Kritchenkov et al. (2020) evidenciaram que o componente triazol betaína quitosana altamente antibacteriano pode ser incorporado na matriz de sódio de succinil quitosana por simples mistura para produzir filmes compactos e uniformes. Nesta pesquisa, as aplicações de teste das películas de mistura como revestimentos de alimentos para bananas reduziram sua perda de peso, perda de vitamina $\mathrm{C}$ e taxa de respiração, e isso resultou no aumento da vida útil das bananas revestidas pelos filmes, sinalizando ser promissor para uso alimentício. Crizel et al. (2018) ponderaram o efeito da adição de diferentes concentrações de farinha e micropartículas de bagaço de azeitona em filmes à base de quitosana. Os autores verificaram que os filmes a base de quitosana com adição de $10 \%$ de micropartículas de bagaço de azeitona eram mais homogêneos e compactos, além de maior resistência mecânica, sem apresentar alterações na permeabilidade e solubilidade ao vapor d'água. Além disso, a adição de micropartículas melhorou as propriedades ópticas e aumentou a ação antioxidante contra a oxidação das nozes durante o período de 31 dias.

Nanoemulsões de quitosana como revestimentos comestíveis para proteção de frutas e alimentos vegetais tem sido bastante investigados nos últimos anos (CHAUDHARY et al., 2020), visando melhorar as funcionalidades desses filmes comestíveis ativos e preservar esses 
alimentos por um período de tempo maior (NAIR et al., 2020). Alimentos frescos, tais como os hortifrutigranjeiros e carnes, são produtos de fácil deteriorização devido à sua composição biológica. Guo; Yadav; Jin (2017) analisaram o uso de revestimentos para fins comestíveis antimicrobianos e filmes de microemulsões para reduzir as populações de patógenos de origem alimentar. Os autores empregaram a goma de fibra de milho como um emulsificante com quitosana, obtendo êxito na redução de bactérias Gram- e Gram+, podendo ser utilizado de modo eficaz para a redução da carga patogênica em alimentos, como carnes prontas para consumo e de morangos, por exemplo. Chien; Sheu; Yang (2007) prepararam fatias de manga com soluções aquosas de $0 \%, 0,5 \%, 1 \%$ e $2 \%$ de quitosana e observaram que a aplicação de um revestimento de quitosana foi capaz de prolongar os atributos de qualidade e estender a vida útil dessa fruta. Assim, a quitosana tem sido utilizada como revestimento comestível em uma variedade de alimentos de origem animal e vegetal, tais como os descritos para peitos de frango, ovos, filetes frescos de peixes, frutos de goiaba (Psidium guajava L.), peras e brócolis recém-cortados (SOUZA et al., 2020), entre outros.

\section{Aplicação biotecnológica: alimentos \& bebidas}

A qualidade biológica do alimento pode ser intensificada e/ou melhorada a partir da utilização de quitosana. Sentença corroborada por Huang et al. (2017), quando analisaram o efeito do tratamento com quitosana enriquecida com ácido salicílico no aumento da qualidade físico-química do kiwi chinês (Actinidia chinensis Planch). Segundo os autores, o tratamento com o polímero manteve a textura e a cor, sendo capaz de inibir a perda de umidade e a mudança de acidez, além de contribuir para atrasar a decomposição da vitamina $\mathrm{C}$ (teor máximo de 97,93 mg/kg) e dos sólidos solúveis, tornado-se uma alternativa favorável para melhorar a qualidade dessa fruta.

A quitosana é uma prodigiosa opção na preservação e prolongamento da vida útil de produtos aquáticos, como peixes e frutos do mar, devido sua ação antimicrobiana contra patógenos deteriorantes de origem alimentar, além do que, a ação antioxidante da quitosana protege os produtos alimentícios contra oxidação lipídica (INANLI et al., 2020), principalmente os alimentos produzidos pela indústria de aquicultura. Rhoades; Roller (2000) investigaram a atividade antimicrobiana de quitosanas degradadas e nativas hidrolisadas por degradação oxidativa-redutiva e lisozima contra microrganismos deteriorantes em alimentos e bebidas, a fim de avaliar seu potencial como produto conservante de alimentos e puderam constatar sua eficiência em alimentos de baixo $\mathrm{pH}$, quando aplicada sozinha ou em combinação com outros sistemas conservantes. Também, foi ressaltado pelos autores que os 
constituintes da matriz alimentar têm papel crucial na eficácia antimicrobiana da quitosana.

Darwesh et al. (2018) produziram nanoquitosana a partir de resíduos de camarão e de biomassa produzida por fungos (Rhizopus stolonifer). Os atores testaram nove grupos de ratos tratados com quitosana ou nanoquitosana de ambas as fontes a 100 e $200 \mathrm{mg} \cdot \mathrm{kg}^{-1}$. Os autores constataram que a adição de quitosana na dieta de todos os grupos não demonstrou alterações significativas nos parâmetros bioquímicos sanguíneos e de estresse oxidativo, quando da comparação ao grupo controle, dados apoiados pela histopatologia do fígado, rim e estômago. Os autores também observaram a ausência de sinais inflamatórios, fibroses ou cirrose nessas no fígado, rim e estômago. Além disso, tanto a quitosana quanto a nanoquitosana de camarão e a fúngica apresentaram alta capacidade antimicrobiana, além da vantagem de produtos considerados atóxicos, podendo ser utilizados como ingredientes na preparação de alimentos.

Ausar et al. (2001) analisaram a hidrólise de um agregado de leite induzido por quitosana, usando enzimas gástricas (pepsina) e intestinais (tripsina e lipase pancreática). Segundo os autores, foi observada inibição progressiva da lipase pancreática em proporção ao aumento da massa molecular da quitosana empregada para induzir a formação do agregado. Ainda, a presença de quitosana afetou a velocidade inicial da reação e reduziu sua extensão. Os resultados sugerem que um agregado de leite induzido por quitosana é bem digerido por proteases gástricas e intestinais, facilitando seu uso oral como componente nutracêutico.

Bough; Landes (1976) demonstraram através de seus estudos que a quitosana, produzida a partir da quitina extraída de resíduos de camarões e caranguejos, era capaz de coagular sólidos suspensos no soro de queijo com a mesma eficácia ou mais do que dez polímeros sintéticos comerciais; enquanto que Oh et al. (2001) averiguaram as atividades antimicrobianas das quitosanas contra quatro espécies de microrganismos deteriorantes de alimentos (Lactobacillus plantarum, Lactobacillus fructivorans, Serratia liquefaciens e Zygosaccharomyces bailii) em meio líquido e maionese. Os autores relataram que o efeito biocida inicial das quitosanas e que as contagens de células viáveis foram expressivamente reduzidas, indicando a viabilidade de uso desse polímero na indústria alimentícia. Savant; Torres (2000) produziram complexos com quitosana e avaliaram quanto ao seu potencial coagulante para o tratamento de soro de queijo Cheddar. O estudo demonstrou com sucesso a eficácia dos complexos com quitosana na floculação de resíduos sólidos suspensos em soro de queijo com mais de $70 \%$ de recuperação de proteína.

Aditivos em alimentos e bebidas devem resistir às condições fisiológicas corporais. Para exercer efeitos benéficos sobre os probióticos, eles devem ser capazes de tolerar as condições do trato gastrintestinal sob as condições ácidas do ambiente estomacal e da bile no 
intestino delgado. O microencapsulamento tem recebido especial atenção, uma vez que pode proteger organismos probióticos contra um ambiente desfavorável e permitir sua liberação em um estado viável e metabolicamente ativo no intestino (CHEN et al., 2015). Shu et al. (2017) microencapsularam Lactobacillus acidophilus em complexo polieletrólito formado por géis de xantana-quitosana e xantana-quitosana-xantana no iogurte, obtendo uma boa sobrevivência da bactéria durante o período de armazenamento (21 dias). Utilizando a mesma bactéria e o mesmo complexo, Shu et al. (2018) avaliaram os efeitos da microencapsulação nas mudanças na sobrevivência e no comportamento de liberação do Lactobacillus acidophilus durante a exposição ao fluido gástrico e intestinal simulados. Os autores observaram que a bactéria encapsulada foi capaz de apresentar alta resistência aos fluidos testados do que amostras não encapsulada. Além disso, a viabilidade de células livres e imobilizadas do Lactobacillus acidophilus incorporada em bebidas lácteas foi avaliada por um período de 21 dias e que a contagem de bactérias viáveis também foi considerada alta.

Pourjafar et al. (2020) aplicaram a quitosana para revestir probióticos de Lactobacilos encapsulados num tipo de bebida iraniana (Doogh) e avaliaram a capacidade de sobrevivência das bactérias e as características do produto final durante o período de 42 dias, tendo sido observado um aumento da capacidade de sobrevivência desses probióticos (Lactobacillus acidophilus e Lactobacillus rhamnosus) na Doogh, como também no lúmen gastrointestinal. Singh et al. (2020) trataram videiras com quitosana $(0,01 \%$ em ácido acético 0,01\%), enquanto as videiras controle foram pulverizadas apenas com ácido acético 0,01\%. Segundo os autores, várias antocianinas monoméricas aumentaram significativamente nas peles dos frutos após o tratamento com quitosana. Esses dados foram corroborados por Silva et al. (2020) quando observaram maior atividade antioxidante e antibacteriana em videiras tratadas com quitosana. Taillandier et al. (2015) investigaram os mecanismos de ação de uma preparação específica de quitosana de origem fúngica sobre Brettanomyces bruxellensis, uma levedura responsável pela produção de compostos aromáticos indesejáveis (fenóis voláteis) em bebidas, alterando a qualidade do vinho. O efeito de inativação foi confirmado pelos autores, além da observação da formação de agregados celulares.

Roller; Covill (1999) investigaram as propriedades antifúngicas da quitosana em meios de laboratório e suco de maçã contra 15 leveduras e bolores associados à deterioração de alimentos, a fim de avaliar o potencial do uso da quitosana como conservante natural. Das cepas de fungos filamentosos testados, a quitosana reduziu com a menor concentração utilizada a taxa de crescimento de Mucor racemosus. O efeito inicial da quitosana no suco de maçã foi biocida. Também avaliando a deteriorização do suco de maçã, Kiskó; Sharp; Roller 
(2015) averiguaram o uso da quitosana para reduzir a ação microbiana sobre esse produto. Os autores isolaram e identificaram microrganismos presentes nos sucos tratados ou não com quitosana. Foi reportado que a quitosana foi capaz de inativar as leveduras de deterioração, mas aumentou a sobrevivência de Escherichia coli O157:H7. A quitosana (0,05-0,1\%) foi capaz de atrasar a deterioração do suco de maçã por leveduras (Kloeckera apiculata e Metschnikowia pulcherrima) e crescimento lento de Saccharomyces cerevisiae e Pichia spp.

Em sistemas vegetais, a quitosana tem sido utilizada para a síntese de nanopartículas visando o sistema de entrega para fertilizantes, herbicidas, pesticidas e micronutrientes para a promoção do crescimento da cultura por meio de uma nutrição equilibrada e sustentada. Além disso, as nanopartículas de quitosana oferecem material genético para a transformação de plantas com segurança (MALERBA; CERANA, 2016), sem afetar os micróbios benéficos do solo. Ainda, é útil para reduzir as perdas de fertilizantes devido à sua capacidade de revestimento que é importante para manter a poluição ambiental sob controle (SHARIF et al., 2018).

O tratamento com quitosana estimula a taxa fotossintética, o fechamento estomático, aumenta as enzimas antioxidantes através das vias de sinalização do óxido nítrico e do peróxido de hidrogênio e induz a produção de ácidos orgânicos, açúcares, aminoácidos e outros metabólitos que são necessárias para os ajustes osmóticos, sinalizações de estresse e metabolismo energético. É utilizada como ferramenta para fitorremediação e biorremediação de solos. Além disso, é empregado como composto antitranspirante por via foliar em muitas plantas, reduzindo o uso de água e garantindo proteção contra outros efeitos negativos (HIDANGMAYUM et al., 2019). Com base nessas propriedades, há um grande interesse no uso de biopolímeros de quitosana em sistemas agrícolas (SHARIF et al., 2018). Savard et al. (2002) avaliaram as propriedades antimicrobianas de polímeros com diferentes massas moleculares de quitosana-lactato contra duas leveduras isoladas de vegetais fermentados e contra três bactérias ácido lácticas de um iniciador misto para chucrute. Os autores puderam constatar que o polímero quitosana-lactato reduziu o crescimento de todos os microrganismos em meio sólido e meio líquido.

Rahman et al. (2018) investigaram os efeitos da aplicação de quitosana sobre o crescimento, produção de frutos e atividades antioxidantes de morangos. Segundo os autores, a aplicação da quitosana contribuiu para o crescimento da planta e a produção de frutos (até $42 \%$ mais) em comparação com o controle não tratado. Os frutos das plantas pulverizadas com quitosana também apresentaram teores expressivamente maiores (até 2,6 vezes) de compostos carotenóides, antocianinas, flavonóides e fenólicos em comparação com o controle 
não tratado. As atividades antioxidantes totais em frutos de plantas tratadas com quitosana também foram maiores (até 2 vezes) quando confrontadas com o grupo sem tratamento. Esses dados vão de encontro com os descritos por He et al. (2018) quando trataram morangos com oligossacarídeos de quitosana e puderam observar uma melhora da qualidade e da capacidade oxidante dessa fruta. Assim, de acordo com o descrito na literatura científica, a quitosana tem se tornando uma excelente opção para uso em vegetais voltados para a produção de alimentos.

\section{Considerações Finais}

Esta mini-revisão se propôs a elencar as principais aplicações biotecnológicas da quitosana, um copolímero obtido a partir da quitina e amplamente empregado nos diferentes segmentos industriais; tendo este trabalho enfocado na inserção desse polímero na fabricação de produtos de revestimento/filmes para embalagens, na aplicação em alimentos de origem animal e de frutas e vegetais, além de bebidas, muitas com funções nutracêuticas. O volume de publicações a respeito do tema é grande e ainda tendência é de crescimento devido às inúmeras possibilidades de associação dos polímeros de quitosana com outros polímeros, principalmente de origem aquática, uma prática quem vem sendo utilizada com sucesso pelos profissionais de biotecnologia para suprir aspectos deficientes da quitosana. Ainda, visando redução de custos operacionais, as fontes aquáticas marinhas têm recebido atenção por apresentar funções biológicas que são altamente desejáveis pela indústria nutracêutica. Assim, são incentivadas novas investigações que possam atrelar esse biopolímero a novos compostos visando à produção de embalagens, alimentos e/ou bebidas suplementadas com quitosana.

\section{Referências}

ANDRADE, M.R. et al. Effect of Cellulose Nanocrystals from Different Lignocellulosic Residues to Chitosan/Glycerol Films. Polymers, v.11, n.4, 658, 2019. https://doi.org/10.3390/polym11040658

AGUILAR, A. et al. Application of Chitosan in Bone and Dental Engineering. Molecules (Basel, Switzerland), v.24, n.16, 3009, 2019. https://doi.org/10.3390/molecules24163009

AHMED, S.; ANNU; ALI, A.; SHEIKH, J. A review on chitosan centred scaffolds and their applications in tissue engineering. International Journal of Biological Macromolecules, v.116, p.849-862, 2018.

AKBARI-ALAVIJEH, S.; SHADDEL, R.; JAFARI, S.M. Encapsulation of food bioactives and nutraceuticals by various chitosan-based nanocarriers. Food Hydrocolloids, v.105, 105774, 2020. doi:10.1016/j.foodhyd.2020.105774 
ALI, A.; AHMED, S. A review on chitosan and its nanocomposites in drug delivery. International Journal of Biological Macromolecules, v.109, p.273-286, 2018.

AHSAN, S.M. et al. Chitosan as biomaterial in drug delivery and tissue engineering. International Journal of Biological Macromolecules, v.110, p.97-109, 2018.

AUSAR, S.F. et al. Hydrolysis of a chitosan-induced milk aggregate by pepsin, trypsin and pancreatic lipase. Bioscience, Biotechnology, and Biochemistry, v.65, n.11, p.2412-2418, 2001. https://doi.org/10.1271/bbb.65.2412

BABU, A.; RAMESH, R. Multifaceted Applications of Chitosan in Cancer Drug Delivery and Therapy. Marine drugs, v.15, n.4, 96, 2017. https://doi.org/10.3390/md15040096

BHAT, I.A. et al. Fabrication and characterization of chitosan conjugated eurycomanone nanoparticles: In vivo evaluation of the biodistribution and toxicity in fish. International Journal of Biological Macromolecules, v.112, p.1093-1103, 2018.

BARANWAL, A. et al. Quitosana: Um material de bio-fabricação indiscutível para aplicações em engenharia de tecidos e bio-detecção. International Journal of Biological Macromolecules, v.110, p.110-123, 2018.

BOUGH, W.A.; LANDES, D.R. Recovery and nutritional evaluation of proteinaceous solids separated from whey by coagulation with chitosan. Journal of Dairy Science, v.59, n.11, p.1874-1880, 1976. https://doi.org/10.3168/jds.S0022-0302(76)84456-6

CAO, Y. et al. Recent Advances in Chitosan-Based Carriers for Gene Delivery. Marine drugs, v.17, n.6, 381, 2019. https://doi.org/10.3390/md17060381

CHAUDHARY, S. et al. Chitosan nanoemulsions as advanced edible coatings for fruits and vegetables: Composition, fabrication and developments in last decade. International Journal of Biological Macromolecules, v.152, p.154-170, 2020. doi:10.1016/j.ijbiomac.2020.02.276

CHEBA, B.A. Chitosan: Properties, Modifications and Food Nanobiotechnology. Procedia Manufacturing, v.46, p.652-658, 2020. https://doi.org/10.1016/j.promfg.2020.03.093

CHEN, H. et al. Effect of complexation conditions on microcapsulation of Lactobacillus acidophilus in xanthan-chitosan polyelectrolyte complex gels. Acta scientiarum polonorum. $\begin{array}{lllll}\text { Technologia Alimentaria, } & \text { v.14, } & \text { n. 2015-213, }\end{array}$ https://doi.org/10.17306/J.AFS.2015.3.22

CHEN, B.; LI, J.; BORGENS, R.B. Neuroprotection by chitosan nanoparticles in oxidative stress-mediated injury. BMC research notes, v.11, n.1, 49, 2018. https://doi.org/10.1186/s13104-018-3162-7

CHEUNG, R.C. et al. Chitosan: An Update on Potential Biomedical and Pharmaceutical Applications. Marine Drugs, v.13, n.8, p.5156-5186, 2015.

CHIEN, P.-J.; SHEU, F.; YANG, F.-H. Effects of edible chitosan coating on quality and shelf life of sliced mango fruit. Journal of Food Engineering, v.78, n.1, p.225-229, 2007. doi:10.1016/j.jfoodeng.2005.09.022 
CRIZEL, T.M. et al. Active food packaging prepared with chitosan and olive pomace. Food Hydrocolloids, v.74, p.139-150, 2018.

doi:10.1016/j.foodhyd.2017.08.007

DARWESH, O.M. et al. Bio-evaluation of crustacean and fungal nano-chitosan for applying as food ingredient. Toxicology reports, v.5, p.348-356, 2018. https://doi.org/10.1016/j.toxrep.2018.03.002

DAETZ, R. et al. Clinical response after chitosan microparticle administration and preliminary assessment of efficacy in preventing metritis in lactating dairy cows. Journal of Dairy Science, v.99, n.11, p.8946-8955, 2016. https://doi.org/10.3168/jds.2016-11400

DRAGOSTIN, O.M. et al. New antimicrobial chitosan derivatives for wound dressing applications. Carbohydrate polymers, v.141, p.28-40, 2016.

DRAGOSTIN, O.M. et al. Designing of Chitosan Derivatives Nanoparticles with Antiangiogenic Effect for Cancer Therapy. Nanomaterials, v.10, n.4, 698, 2020.

DUTTA, J.; TRIPATHI, S.; DUTTA, P.K. Progress in antimicrobial activities of chitin, chitosan and its oligosaccharides: a systematic study needs for food applications. Food science and technology international $=$ Ciencia $y$ tecnologia de los alimentos internacional, v.18, n.1, p.3-34, 2012. https://doi.org/10.1177/1082013211399195

ELSABEE, M.Z.; ABDOU, E.S. Chitosan based edible films and coatings: A review. Materials Science and Engineering: C, v.33, n.4, p.1819-1841, 2013.

http://dx.doi.org/10.1016/j.msec.2013.01.010

EL KNIDRI, H. et al. Extraction, chemical modification and characterization of chitin and chitosan: A review. International Journal of Biological Macromolecules, v.109, p.273-286, 2018.

ELIEH-ALI-KOMI, D.; HAMBLIN, M.R. Chitin and Chitosan: Production and Application of Versatile Biomedical Nanomaterials. International Journal of Biological Macromolecules, v.4, n.3, p.411-427, 2016.

FAN, X. et al. Development of tilapia collagen and chitosan composite hydrogels for nanobody delivery. Colloids and Surfaces B: Biointerfaces, v.195, 111261, 2020. doi:10.1016/j.colsurfb.2020.111261

FARIAS, B.S. et al. Chitosan-functionalized nanofibers: A comprehensive review on challenges and prospects for food applications. International Journal of Biological Macromolecules, v.123, p.210-220, 2019. https://doi.org/10.1016/j.ijbiomac.2018.11.042

FONSECA-SANTOS, B.; CHORILLI, M. An overview of carboxymethyl derivatives of chitosan: Their use as biomaterials and drug delivery systems. Materials Science Engineering. C, Materials for biological applications, v.77, p.1349-1362, 2017. 
GAIHRE, B.; LECKA-CZERNIK, B.; JAYASURIYA, A.C. Injectable nanosilica-chitosan microparticles for bone regeneration applications. Journal of Biomaterials Applications, v.32, n.6, p.813-825, 2018. https://doi.org/10.1177/0885328217741523

GUO, M.; YADAV, M.P.; JIN, T.Z. Antimicrobial edible coatings and films from microemulsions and their food applications. International Journal of Food Microbiology, v.263, p.9-16, 2017. doi:10.1016/j.ijfoodmicro.2017.10.002

HAMIL, S. et al. Use of sea urchin spines with chitosan gel for biodegradable film production. International Journal of Biological Macromolecules, v.152, p.102-108, 2020. doi:10.1016/j.ijbiomac.2020.02.263

HE, Y. et al. Pre-Harvest Treatment of Chitosan Oligosaccharides Improved Strawberry Fruit Quality. International Journal of Molecular Sciences, v.19, n.8, 2194, 2018. https://doi.org/10.3390/ijms19082194

HIDANGMAYUM, A. et al. Application of chitosan on plant responses with special reference to abiotic stress. Physiology and molecular biology of plants: an international $\begin{array}{llllll}\text { journal of functional plant biology, v.25, n.2, p.313-326, } 2019 . & \end{array}$ https://doi.org/10.1007/s12298-018-0633-1

HU, Z. et al. Chitosan-Based Composite Materials for Prospective Hemostatic Applications. Marine Drugs, v.16, n.8, 273, 2018.

HUANG, Z. et al. Physicochemical properties enhancement of Chinese kiwi fruit (Actinidia chinensis Planch) via chitosan coating enriched with salicylic acid treatment. Journal of Food Measurement and Characterization, v.11, n.1, p.184-191, 2017. https://doi.org/10.1007/s11694-016-9385-1

IACOB, A.T. et al. Preparation, Characterization and Wound Healing Effects of New Membranes Based on Chitosan, Hyaluronic Acid and Arginine Derivatives. Polymers, v10, n.6, 607, 2018.

INANLI, A.G. et al. The impact of chitosan on seafood quality and human health: A review. Trends in Food Science \& Technology, v.97, p.404-416, 2020. https://doi.org/10.1016/j.tifs.2020.01.029

KASHYAP, P.L.; XIANG, X.; HEIDEN, P. Chitosan nanoparticle based delivery systems for sustainable agriculture. International Journal of Biological Macromolecules, v.77, p.3651, 2015. doi:10.1016/j.ijbiomac.2015.02.039

KRAVANJA, G. et al. Chitosan-based (Nano)materials for Novel Biomedical Applications. Molecules, v.24, n.10, 1960, 2019.

KIDIBULE, P.E. et al. Use of chitin and chitosan to produce new chitooligosaccharides by chitinase Chit42: enzymatic activity and structural basis of protein specificity. Microbial Cell Factories, v.17, n.1, 47, 2018. https://doi.org/10.1186/s12934-018-0895-x 
KISKÓ, G.; SHARP, R.; ROLLER, S. Chitosan inactivates spoilage yeasts but enhances survival of Escherichia coli O157:H7 in apple juice. Journal of Applied Microbiology, v.98, n.4, p.872-880, 2005. https://doi.org/10.1111/j.1365-2672.2004.02527.x

KRITCHENKOV, A.S. et al. Active antibacterial food coatings based on blends of succinyl chitosan and triazole betaine chitosan derivatives. Food Packaging and Shelf Life, v.25, 100534, 2020. doi:10.1016/j.fpsl.2020.100534

LANCINA, M.G.; SHANKAR, R.K.; YANG, H. Chitosan nanofibers for transbuccal insulin delivery. Journal of biomedical materials research. Part A, v.105, n.5, p.1252-1259, 2017. https://doi.org/10.1002/jbm.a.35984

LIU, W. et al. Synthesis, characterization and antifungal efficacy of chitosan derivatives with triple quaternary ammonium groups. International Journal of Biological Macromolecules, v.114, p.942-949, 2018.

MALERBA, M.; CERANA, R. Chitosan Effects on Plant Systems. International Journal of Molecular Sciences, v.17, n.7, 996, 2016. https://doi.org/10.3390/ijms17070996

MALIK, A. et al. Novel application of trimethyl chitosan as an adjuvant in vaccine delivery. International Journal of Nanomedicine, v.13, p.7959-7970, 2018. https://doi.org/10.2147/IJN.S165876

MANIGANDAN, V. et al. Chitosan Applications in Food Industry. In: Biopolymers for Food Design, p.469-491, 2018. doi:10.1016/b978-0-12-811449-0.00015-3

MARTĂU, G.A.; MIHAI, M.; VODNAR, D.C. The Use of Chitosan, Alginate, and Pectin in the Biomedical and Food Sector-Biocompatibility, Bioadhesiveness, and Biodegradability. Polymers, v.11, n.11, 1837, 2019. https://doi.org/10.3390/polym11111837

MATICA, M.A. et al. Chitosan as a Wound Dressing Starting Material: Antimicrobial Properties and Mode of Action. International Journal of Molecular Sciences, v.20, n.23, 5889, 2019.

MU, M.; LI, X.; TONG, A.; GUO, G. Multi-functional chitosan-based smart hydrogels mediated biomedical application. Expert Opinion on Drug Delivery, v.16, n.3, p.239-250, 2019.

MUANPRASAT, C.; CHATSUDTHIPONG, V. Chitosan oligosaccharide: Biological activities and potential therapeutic applications. Pharmacology \& Therapeutics, v.170, p.80-97, 2017.

MUJTABA, M. et al. Current advancements in chitosan-based film production for food technology; A review. International Journal of Biological Macromolecules, v.121, p.889904, 2018. doi:10.1016/j.ijbiomac.2018.10.109

MUXIKA, A. et al. Chitosan as a bioactive polymer: Processing, properties and applications. International Journal of Biological Macromolecules, v.105, p.1358-1368, 2017. 
NAIR, M.S. et al. Enhancing the functionality of chitosan- and alginate-based active edible coatings/films for the preservation of fruits and vegetables: A review. International Journal of Biological Macromolecules, v.164, p.304-320, 2020. doi:10.1016/j.ijbiomac.2020.07.083

$\mathrm{OH}$, H.I. et al. Antimicrobial characteristics of chitosans against food spoilage microorganisms in liquid media and mayonnaise. Bioscience, Biotechnology, and Biochemistry, v.65, n.11, p.2378-2383, 2001. https://doi.org/10.1271/bbb.65.2378

Olatunji, O. Aquatic Biopolymers Understanding their Industrial Significance and Environmental Implications, 1st ed., Springer International Publishing, 2020. doi:10.1007/978-3-030-34709-3_16.

OLIVEIRA, E.B. et al. Using chitosan microparticles to treat metritis in lactating dairy cows. Journal of Dairy Science, v.103, n.8, p.7377-7391, 2020. https://doi.org/10.3168/jds.201918028

OLIVEIRA, V.M. et al. Physical, biochemical, densitometric and spectroscopic techniques for characterization collagen from alternative sources: a review based on the sustainable valorization of aquatic by-products. Journal of Molecular Structure, v.1224, 129023, 2021. https://doi.org/10.1016/j.molstruc.2020.129023

OTTONELLI, M. et al. Chitosan-Stabilized Noble Metal Nanoparticles: Study of their Shape Evolution and Post-Functionalization Properties. Nanomaterials, v.10, n.2, 224, 2020.

PAUL, P.; KOLESINSKA, B.; SUJKA, W. Chitosan and Its Derivatives - Biomaterials with Diverse Biological Activity for Manifold Applications. Mini-Reviews in Medicinal Chemistry, v.19, n.9, p.737-750, 2019.

PATRULEA, V. et al. Chitosan as a starting material for wound healing applications. European journal of pharmaceutics and biopharmaceutics, v.97, p.417-426, 2015.

PEREDA, M. et al. Polyelectrolyte films based on chitosan/olive oil and reinforced with cellulose nanocrystals. Carbohydrate polymers, v.101, p.1018-1026, 2014. https://doi.org/10.1016/j.carbpol.2013.10.046

POURJAFAR, $\mathrm{H}$. et al. Viability of microencapsulated and non-microencapsulated Lactobacilli in a commercial beverage. Biotechnology Reports, v.25, e00432, 2020. https://doi.org/10.1016/j.btre.2020.e00432

PRIYADARSHI, R.; RHIM, J.-W. Chitosan-based biodegradable functional films for food packaging applications. Innovative Food Science \& Emerging Technologies, v.62, 102346, 2020. doi:10.1016/j.ifset.2020.102346

RAHMAN, M. et al. Chitosan biopolymer promotes yield and stimulates accumulation of antioxidants in strawberry fruit. PloS one, v.13, n.9, e0203769, 2018. https://doi.org/10.1371/journal.pone.0203769

REN, L. et al. Influence of chitosan concentration on mechanical and barrier properties of corn starch/chitosan films. International Journal of Biological Macromolecules, v.105, p.1636-1643, 2017. 
https://doi.org/10.1016/j.ijbiomac.2017.02.008

RHOADES; ROLLER, S. Antimicrobial actions of degraded and native chitosan against spoilage organisms in laboratory media and foods. Applied and Environmental Microbiology, v.66, n.1, p.80-86, 2000. https://doi.org/10.1128/aem.66.1.80-86.2000

ROCHA, M.A.M.; COIMBRA, M.A.; NUNES, C. Applications of chitosan and their derivatives in beverages: a critical review. Current Opinion in Food Science, v.15, p.61-69, 2017. doi:10.1016/j.cofs.2017.06.008

ROLLER, S.; COVILL, N. The antifungal properties of chitosan in laboratory media and apple juice. International Journal of Food Microbiology, v.47, n.1-2, p.67-77, 1999. https://doi.org/10.1016/s0168-1605(99)00006-9

ROZMAN, N. et al. Potential Antimicrobial Applications of Chitosan Nanoparticles (ChNP). Journal of Microbiology and Biotechnology, v.29, n.7, p.1009-1013, 2019. https://doi.org/10.4014/jmb.1904.04065

SAMAYANPAULRAJ, V. et al. Identification and characterization of virulent Aeromonas hydrophila Ah17 from infected Channa striata in river Cauvery and in vitro evaluation of shrimp chitosan. Food Science \& Nutrition, v.8, n.2, p.1272-1283, 2020. https://doi.org/10.1002/fsn3.1416

SATO, T. et al. Chitosan-Based Coacervate Polymers for Propolis Encapsulation: Release and Cytotoxicity Studies. International Journal of Molecular Sciences, v.21, n.12, p.4561, 2020.

SARODE, S. et al. Overview of wastewater treatment methods with special focus on biopolymer chitin-chitosan. International Journal of Biological Macromolecules, v.121, p.1086-1100, 2019. doi:10.1016/j.ijbiomac.2018.10.089

SAVANT, V.D.; TORRES, J.A. Chitosan-based coagulating agents for treatment of cheddar cheese whey. Biotechnology Progress, v.16, n.6, p.1091-1097, 2000.

SAVARD, T.; et al. Antimicrobial action of hydrolyzed chitosan against spoilage yeasts and lactic acid bacteria of fermented vegetables. Journal of Food Protection, v.65, n.5, p.828$833,2002$.

SCHMITZ, C. et al. Conversion of Chitin to Defined Chitosan Oligomers: Current Status and Future Prospects. Marine drugs, v.17, n.8, 452, 2019. https://doi.org/10.3390/md17080452

SHARIF, R. et al. The Multifunctional Role of Chitosan in Horticultural Crops; A Review. Molecules (Basel, Switzerland), v.23, n.4, 872, 2018.

SHU, G. et al. Microencapsulation of Lactobacillus Acidophilus by Xanthan-Chitosan and Its Stability in Yoghurt. Polymers, v.9, n.12, 733, 2017. https://doi.org/10.3390/polym9120733

SHU, G. et al. Effect of Xanthan ${ }^{-}$Chitosan Microencapsulation on the Survival of Lactobacillus acidophilus in Simulated Gastrointestinal Fluid and Dairy Beverage. Polymers, v.10, n.6, 588, 2018. https://doi.org/10.3390/polym10060588 
SHARIATINIA, Z. Pharmaceutical applications of chitosan. Advances in Colloid and Interface Science, v.263, p.131-194, 2018.

SHARIATINIA Z. Pharmaceutical applications of chitosan. Advances in colloid and interface science, v.263, p.131-194, 2019.

SINGH, R.K. et al. Chitosan Application in Vineyards (Vitis vinifera L. cv. Tinto Cão) Induces Accumulation of Anthocyanins and Other Phenolics in Berries, Mediated by Modifications in the Transcription of Secondary Metabolism Genes. International Journal of Molecular Sciences, v.21, n.1, 306, 2020. https://doi.org/10.3390/ijms21010306

SONIN, D. et al. Biological Safety and Biodistribution of Chitosan Nanoparticles. Nanomaterials, v.10, n.4, 810, 2020.

SONG, Z.; LI, G.; GUAN, F.; LIU, W. Application of Chitin/Chitosan and Their Derivatives in the Papermaking Industry. Polymers, v.10, n.4, 389, 2018. https://doi.org/10.3390/polym10040389

SOUZA, V. et al. Chitosan Composites in Packaging Industry-Current Trends and Future Challenges. Polymers, v.12, n.2, 417, 2020. https://doi.org/10.3390/polym12020417

SILVA, V. et al. Comparative Insight upon Chitosan Solution and Chitosan Nanoparticles Application on the Phenolic Content, Antioxidant and Antimicrobial Activities of Individual Grape Components of Sousão Variety. Antioxidants, v.9, n.2, 178, 2020. https://doi.org/10.3390/antiox9020178

SUSENO, N. et al. Improving Shelf-life of Cavendish Banana Using Chitosan Edible Coating. Procedia Chemistry, v.9, p.113-120, 2014. doi:10.1016/j.proche.2014.05.014

TAILLANDIER, P. et al. Effect of a fungal chitosan preparation on Brettanomyces bruxellensis, a wine contaminant. Journal of Applied Microbiology, v.118, n.1, p.123-131, 2015. https://doi.org/10.1111/jam.12682

VAN DEN BROEK, L.A. et al. Chitosan films and blends for packaging material. Carbohydrate polymers, v.116, p.237-242, 2015.

https://doi.org/10.1016/j.carbpol.2014.07.039

VERLEE, A.; MINCKE, S.; STEVENS, C.V. Recent developments in antibacterial and antifungal chitosan and its derivatives. Carbohydrate Polymers, v.164, p.268-283, 2017. doi:10.1016/j.carbpol.2017.02.001

WANG, H.; QIAN, J.; DING, F. Emerging Chitosan-Based Films for Food Packaging Applications. Journal of agricultural and food chemistry, v.66, n.2, p.395-413, 2018. https://doi.org/10.1021/acs.jafc.7b04528

WANG, J. et al. Beneficial Metabolic Effects of Chitosan and Chitosan Oligosaccharide on Epididymal WAT Browning and Thermogenesis in Obese Rats. Molecules (Basel, Switzerland), v.24, n.24, 4455, 2019. https://doi.org/10.3390/molecules24244455 
WANG, W. et al. Chitosan Derivatives and Their Application in Biomedicine. International Journal of Molecular Sciences, v.21, n.2, 487, 2020.

WANG, Z.F. et al. Therapeutic effect of chitosan on CCl4- induced hepatic fibrosis in rats. Molecular medicine reports, v.18, n.3, p.3211-3218, 2018. https://doi.org/10.3892/mmr.2018.9343

XU, Y. et al. Injectable and Self-Healing Chitosan Hydrogel Based on Imine Bonds: Design and Therapeutic Applications. Journal Molecular Sciences, v.19, n.8, 2198, 2018. https://doi.org/10.3390/ijms19082198

YOUNES, I.; et al. Chitin and chitosan preparation from shrimp shells using optimized enzymatic deproteinization. Process Biochemistry, v.47, n.12, p.2032-2039, 2012.

ZARGAR, V.; ASGHARI, M.; DASHTI, A. A Review on Chitin and Chitosan Polymers: Structure, Chemistry, Solubility, Derivatives, and Applications. ChemBioEng Reviews, v.2, n.3, p.204-226, 2015. doi:10.1002/cben.201400025

ZHAO, D. et al. Biomedical Applications of Chitosan and Its Derivative Nanoparticles. Polymers, v.10, n.4, 462, 2018. 